International Journal of Engineering \& Technology, $7(2.32)(2018) 224-227$
International Journal of Engineering \& Technology
Website: www.sciencepubco.com/index.php/IJET
Research paper

\title{
Data Management in IOT Applications
}

\author{
Dr .Sanakkayala SatyaNarayana ${ }^{1}$,G.V. Sai Bharath ${ }^{2}$,Katakam Sri Lakshmi Sahithi ${ }^{3}$,Adusumilli Sai Rutwik ${ }^{4}$ \\ ${ }^{1,2,3,4}$ Department of CSE, K L E F, Vaddeswaram, Guntur, Andhra Pradesh, India- 522502. \\ *Corresponding author E-mail: s.satyans1 @ gmail.com
}

\begin{abstract}
With the technology leaping towards a new phase the next big that is happening is IOT and managing the huge amount of data that is being produced. To apprehend the real Internet of Things in which the entirely is interconnected, direct interactions between sensors and actuators, also known as bindings, are essential. As more and more devices are getting connected to the internet there is a lot of data that is being generated. We need to maintain the quality of data and it should be manageable for future use. Consequently, in evaluation to subsisting studies on smart cities we give a information driven edge depicting the central information administration methodologies employed to check consistency, interoperability, granularity and re-convenience of the information created by strategies for the fundamental Internet of Things( IoT) for smart cities. We try to find the proper communication between the devices and finally try to implement the details for a system. In this paper we are trying to do survey on how the large amount of data is being stored and various strategies for handling the data by using some architectures for the smart traffic system. We are trying to use the SWIFT architecture for analyzing the traffic in smart cities.
\end{abstract}

Keywords:IOT, management of data, heterogeneity, SWIFT architecture.

\section{Introduction}

With the current fashion heading towards ubiquitous computing, accumulating and using data every day for the numerous things around us has contributed significantly to the Internet of Things (IoT). IoT has technology blending into our daily lives displaying the tremendous research shift in finding approaches to process data in the most efficient and secure way. By 2050 it is expected that 70 percent of the population will be residing in cities, it is predicted that metropolis will face diverse demanding situations from manageability and use to protection and capable administration transport. Late advances inside the compelling incorporation of organized information frameworks, detecting and specialized devices, information sources, primary management, and physical basis are making new possibilities to reduce activity clog, warfare wrongdoing. Domestic financial development, decrease nursery gasses, and make close by governments greater open, responsive, and effective. An ever-increasing number of urban communities are beginning to outfit the power of sensors, associated with natives provided with cell phones, distributed computing, fast frameworks, and information investigation.

We contend that internet of factors can possibly supply a unavoidable machine of related devices and sensors for SCC, and extensive data research can likely empower the pass from IoT to nonstop manipulate wanted for SCC. The reason for this paper is to symbolize SCC, display openings and demanding situations of IoT and huge information investigation in SCC, and exhibits the utilization of IoT and huge information examination in SCC. The myriad sensors and actuators, but, preserve producing massive quantities of information each second. Sitting on hills of undiscovered information, smart metropolis are plausible to unearth the enormous undertaking of mining believable data from information being produced over the keen city. But, to deal with such voluminous amounts data, the conventional information handling proce- dures strategies might not be good enough. To recover from this difficulty, we present standards of Big information investigation for handling enormous information engendered in smart metropolis for its capability software in numerous fields like shrewd framework, savvy condition, keen power and vitality, keen activity, shrewd wellness administrations, savvy squander, and so on. To demonstrate the utilize of these standards, we advice few strategies to deal with large information for good traffic management in smart metropolis.

\section{Background Literature}

A metropolis may be referred 'smart' at the same time as interests in human \& social capital and present day data \& discussion framework, gasoline sustainable financial magnification for higher pleasant of existence, through savvy administration of herbal assets and participatory administration. This ICT revolution which fuelled the increase of urban communities, permits unparalleled technology of information, in the meantime as at the identical time offering pervasive open get admission to data. The worldwide smart city commercial center is ready to develop a more prominent than $14 \%$, and is anticipated to reach over $\$ 1.3$ trillion by methods for 2019. As digitization has end up being an irreplaceable a piece of social presence, it is miles imagined that around $90 \%$ of the world's digitized information was caught over only the previous 2 years. This has driven numerous legislatures and R\&D business to make utilization of Big information innovation to help the improvement and livability of metropolis cities. Big Data is not always most effective big, but plenty unstructured, complicated, heterogeneous, made out of sundry information sorts spilling information and might be even uncertain, loud or flawed, which may likewise have impact on the factual and information examination strategies and might lessen the overall performance of accuracy on the mining outcomes. Traditional data logical calculations 
might be connected for assessment of Big information in view of the at specific zone space (e.g., classification algorithms inclusive of k-nearest neighbour, linear and quadratic discriminant evaluation, Naive Bayes,.

Huge information is a crucial thing of metropolis urban communities applications as monstrous information created from keen sensors, Internet of Things(IoT), swarm sourced information associations and government to give shrewd answers for savvy city occupants. Huge information is described by three features (3-Vs): volume, velocity and variety. Volume alludes to the extent of information that has been engendered from numerous resources. Velocity is the speed at which information engendered, stored, analysed,handled. Variety alludes to the extraordinary assortments of information being engendered. As extreme information produced unstructured it can't be effortlessly arranged or organized

There are predictions that the IoT makes, so you can easiest happen if there is a money related goal and speculations are made by organizations. Additionally given that access expenses for assembling objects with IoT innovation will be high, certain organization may rule. Facilitate mechanical advances are required to supply vitality productive gadgets and hardware that can be made neatly, included into the common assembling strategies, and reused. There are many promising utility regions, some of that are now valuing the advantages of a restricted IoT, gave through Internet-empowered RFID labels or remote sensor systems. Application regions include: fabricating, store network administration, vitality, wellbeing, care, and protection. But, some of specialized demanding situations stay, which should to be conquer, before the overall IoT imaginative and prescient turns into a reality. A portion of the vital requesting circumstances are versatility, distinguishing proof, and addressability, heterogeneity, and administration ideal models, notwithstanding innovation for security, privateness, believe mechanisms. In this paper we are going to see various classifications, data management techniques in large databases in various IOT applications. We emphasize extra on data management so that we will be capable of draw conclusions for the future references.

\section{Database Issues in the Internet of Things}

\subsection{Size, Scale and Indexing}

The dimensions and scale of the information in the IoT is exceptionally gigantic. Information ought to be controlled by means of dependable nearby clients. Neighbourhood clients will figure out which information administrations are to be made available to the worldwide system. So that, the IoT creations can perform on more than one level: private and open. Clients may join for accessing to certain exclusive information or may, then again, get to information openly accessible over general society Internet. There might be differentiate in nature of information depending on proprietorship and level of care. Well ordered trust and notoriety frameworks will give data to clients on the nature of the information.

In the Worldwide area there can be a requirement for a focal expert, for handling locations and identifiers, as there might be with the present day Internet. Ordering will be a noteworthy test. Finding a thing in a in our current reality where every physical protest have an IP address won't be facile, Except we will devise appropriate ordering techniques. Working in the library list administration would possibly give some guidelines, However the IoT will have specific sorts of items. Making an inventory of everything, is a time taking challenge. A few articles will be openly available, some will require different levels of access control, and some might be private to the proprietor. At first the IoT is probably going to create through nearby frameworks that can be ordered rationally inside a limited area. As neighbourhood frameworks converge with worldwide frameworks, new ordering techniques should be produced.

\subsection{Query Languages}

The current famous enquiry in database frameworks depend on organized information. Organized Query Language (SQL) is the most used case. In the course of the most recent years, there have been recommendations for question dialects for semi-organized information, which is more run of the information hung on the Internet. The IoT will have different sorts of clients: easy-going clients that quickly visit a site to get a few information or data, master clients that know precisely what information they require and where to discover it, and clients that lie some place in the middle. There are various settings a similar individual can be any of these distinctive sorts of clients. It hence appears to be essential that diverse sorts of information get to offices be accessible. Easygoing clients should get to the IoT through an easy to use graphical UI (GUI), with itemized clarification accessible on any protest, and more adaptable, effective, and productive access interfaces will be required for master clients. Administrations can be utilized to give the two sorts of access.

\subsection{Process Modeling and Transactions}

It is far possibly that greatest procedures and provided as organizations at the IoT. Service Oriented Architecture (SOA) is getting to be an essential method for assisting interoperability in electronic frameworks. The focal concept is that autonomous outfits provide administrations in a uniform manner, which different clients would then be capable of soak up. Hence, execution points of interest are escaped the clients of the administrations. Application procedures will regularly be comprised of various lower level exchanges. Exchanges thus will be comprised of lower level operations or administrations. In this manner, the subject of exchange handling in the IoT emerges. Every single partaking site must affirm their availability to confer before the submit order is issued by the organizing site and kept in touch with the database log.

Many factors are concerned in the information administration inside the IoT condition. Some of the most paramount concepts assists us to empower and apprehend the difficulties and possibilities of information administration are:

- Data Accumulation and Analysis

- Big Data

- Semantic Sensor Networking

- Virtual Sensors

Each day the usage of the databases is growing at exponential rates. Meanwhile the need to process and survey the gigantic measure of data for business purposes has in like manner extended dependably. Moreover extending wide variety of affiliations is facing the issue of sudden augmentation and the measure of the databases used as a piece of the present particular world has been creating at exponential rates. Tremendous data is a thought portraying data that has three fundamental uniqueness. To begin with, notable extent of information. Second, the records can't be prepared of time into standard database tables and third, the data is made with large velocity and ought to be gotten and immediately taken care of. Information minimization has uncommon stress over the vast accumulation and overseeing of person data in regime, continue to be solitary, administrative computer databases. The thought used to constrain the gathering and capacity of personal records as to keep capable sodalities from building gigantic dossiers of pure individuals which may want to be utilized for instance, for example, administration, organization, development, and disparity. 
Betokening is, limiting informational collection and ability in stances, would benefit ensure the person opposition security interruptions by the State or other puissant association . Data mining can reinforce decisions in various domains, for example, retail, making, broadcast communications, human administrations , confirmation and delivery. It is utilized to choose new getting designs, perceive illegal consumptions, identify card cheats.

\subsection{Challenges in Handling Big Data for Smart Traffic}

- Identifying the different types of data sources.

- Combining sundry heterogeneous information resources into a cognition model.

- Store the information based on the location (spatially-related data).

- Time related data.

- Data sparseness- as there are numerous areas to deduce, confining the quantity of movement following stations.

Context is a crucial issue of analytic choice process. Context is any realities from data perceptions about elements relying on the interplay between an entity and an application. The meaning of context fluctuates with the vicissitude in implementation condition, i.e., computing surrounding, utilize condition and physical condition. By utilizing context analytics with sizably voluminous data, excessive satisfactory information models can be created and actualized to derive characteristics', patterns, and connection from unstructured data and cognate established road traffic information Contextual information can be habituated to offer higher choices Integrating context with huge data analytics could avail in recognising connection among cognate associated substances crosswise over huge, inadequate, and inconsequential hoards of noteworthy and cutting edge measurements.

\section{SWIFT Architecture for SMART TRAF- FIC}

Here we examine the execution of SWIFT architecture giving an answer for definitely intellective movement administration in smart cities. The whole process is separated into following steps

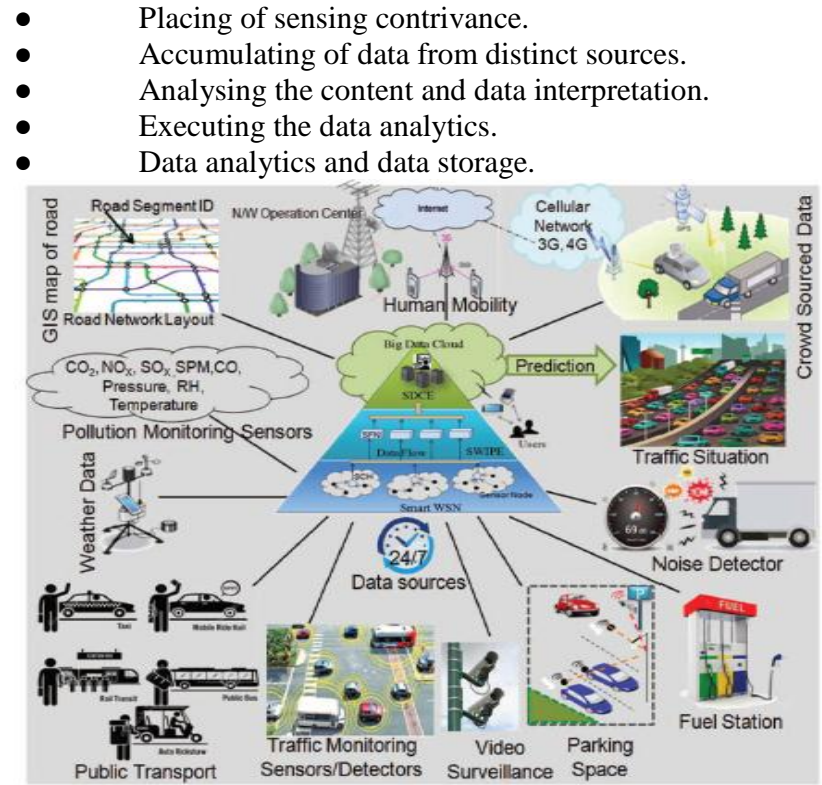

Fig. 1. Big data source for road traffic tracking

For parkway environments, static infrastructure is the most utilizable however for urban street systems where the movement is very factor crosswise over sundry system section at various circumstances of day a zone savvy activity information accumulation is required. For accumulating area particular, fleeting movement information from distinctive assets, the whole street system of a smart city can partitioned into disjoint fragment. Every fragment of the street is intended to have organized path with path train for the vehicles. The SWSN is tangible layer fashioned via Smart Cluster Heads (SCHs)going about as information portions, which files information at standard interims. The street sections has one greater Smart Cluster Heads ( $\mathrm{SCH}(\mathrm{s})$ ), introduced on overhead assembling stage or factor of access and activity checking sensors. The sensors are conveyed on two sides of the street positions for eg activity lampposts, contingent upon the distance of the street and the street is couple-way. The area and approach-particular Smart Cluster Heads (SCHs) total information from all activity checking contrivance and send that movement of data to the closeby Smart Fusion Node. The paramount highlight of the Smart Cluster Heads(SCH) is to perform setting labelling on crude (information change) amassed from the sensors and conduct factual investigation to decide their repute and lifetime, and engender alarms to make a move against flawed hubs. The Smart Cluster Heads stores the approaching spatio-worldly movement information in a period window (time length) engendered at standard interims of time and plays out the going with operations.

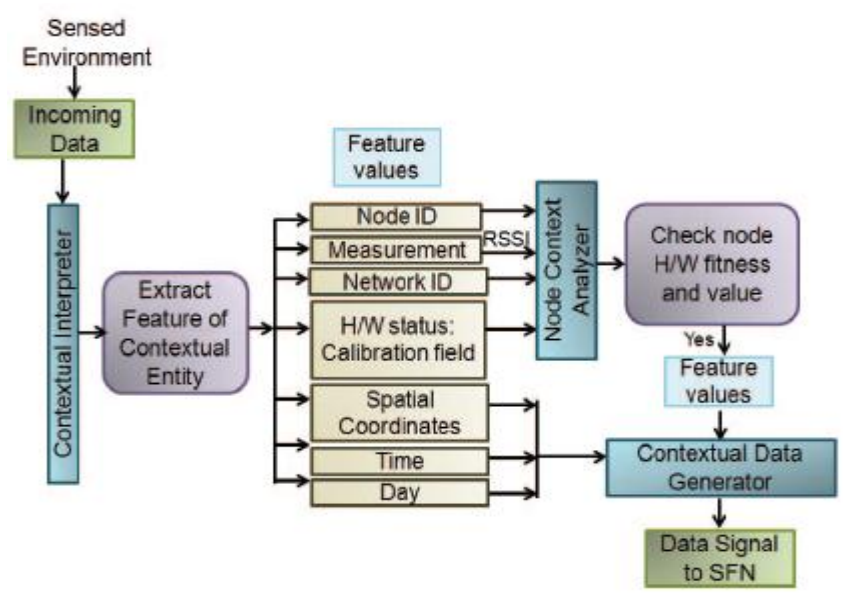

Fig. 2. Rudiment operations executed on contextual data

Based on the data collected we can extract the features along with other measurements. It uses its information to select some training methodologies for decision login and the message can be sent to nearby traffic manipulate points for changing the routes.

\section{Conclusion}

As many sensors are being used these days, immense amount of data is being engendered every second. Smart communities have been raising daily which is leading to the rise of data generated. In this paper we have done a survey on how the huge data has to be broken down for further purposes so that we can use the data more efficiently for future purposes. We have taken the help of SWIFT architecture so that we can analyse the procedure of enormous information cognate to wide range of credible time movement data through sensors. Internet of things has a potential for providing the ubiquitous computing for smart and connected devices. The architecture is adaptable to include various new technologies and policies and help the people for enabling better conditions for living. We can further try to implement this study for traffic applications.

\section{References}

[1] Networking and Information Technology Research and Development(NITRD) 
(2015).SmartandConnectedCommunitiesFramework.[Online].Avail able: https://www.nitrd.gov/sccc/materials/scccframework.pdf.

[2] Y. Sun, Y. Xia, H. Song, and R. Bie, "'Internet of things services for small towns," in Proc. Int. Conf. Identi_cat., Inf. Knowl. Internet Things (IIKI), Oct. 2014, pp. 92_95.

[3] J. Jara, Y. Sun, H. Song, R. Bie, D. Genooud, and Y. Bocchi, 'Internet of Things for cultural heritage of smart cities and smart regions," in Proc.IEEE 29th Int. Conf. Adv. Inf. Netw. Appl.Workshops (WAINA), Mar. 2015,pp. 668_675.

[4] H. Song, R. Srinivasan, T. Sookoor, and S. Jeschke, Smart Cities: Founda- tions and Principles. Hoboken, NJ, USA: Wiley, 2016.

[5] U.S. Department of Transportation. (2013). Livability 101. [Online].

[6] MervatAbu-Elkheir,Mohammad Hayajneh,and,Najah Abu Ali: Data Management for the Internet of Things: Design Primitives and Solution Published online 2013 Nov 14.

[7] A Cognitive Adopted Framework for IoT Big-Data Management and Knowledge Discovery ProspectiveNilamadhab Mishra, Chung-Chih Lin, Hsien-Tsung Chang First Published January 1, 2015

[8] N. D. Milder and A. Dane, "Revitalizing small towns: Resolving downtown challenges," Econ. Develop.J.,2013.[Online]. Available:http://www.ruraldesign.org/resource/revitalizing-small-townsresolving-downtown-challenges.

[9] Annex, "World commission on environment and development," Oxford Univ. Press, New York, NY, USA, 1987. Tech. Rep. A/42/427, 1987.

[10] http://www.softwareadvice.com/resources/iot-data-analytics-usecases/

[11] Approaches and Methods for Data Analytics in IoT written by DAVE WAGSTAFF, JANUARY 2016

[12] Smart cities: A survey on data management, security, enabling technologies written by Ammar Gharaibeh, Member, IEEE, Mohammad A. Salahuddin, Member, IEEE, Sayed J. Hussini, Student Member, IEEE

[13] Strategies to handle Big Data for traffic management in smart cities written by Satyanarayana V Nandury and Beneyaz A Begum.

[14] L. M. A. Bettencourt, "The Uses of Big Data in Cities," Big Data, vol.2, no. 1, pp. 12-22, Mar. 2013.

[15] C. Perera, A. Zaslavsky, P. Christen, and D. Georgakopoulos, "Context aware computing for The Internet of Things: A Survey," IEEE Commun. Surveys Tuts., vol. 16, no. 1, pp. 414-454, Jan. 1, 2014.

[16] G. S. Mann, and A. McCallum, "Generalized expectation criteria for semi-supervised learning with weakly labeled data," J. of Mach. Learning Res., vol. 11, pp. 955-984, Feb. 2010.

[17] A. Blum, and T. Mitchell, "Combining labeled and unlabeled data with co-training," in Proc. 11th Ann. Conf. on Comput.Learning Theory, pp.92-100, 1998.

[18] K. Nigam, and R. Ghani, "Analyzing the effectiveness and applicability of co-training," in Proc. of CIKM, 2000.

[19] Z. H. Zhou, and M. Li, "Tri-training: exploiting unlabeled data using three classifiers," Trans. Knowl. and Data Eng., vol. 17, no. 11, pp. 1529-1541, 2005.

[20] B. Schilit, N. Adams, and R. Want, "Context-Aware Computing Applications," 1st Int. Workshop on Mobile Comput. Syst. and Appl.,1994, pp. 85-90. 2016 Intl. Conference on Advances in Computing, Communications and Informatics (ICACCI), Sept. 21-24, 2016, Jaipur, India. 